\title{
Improving the Quality of Data Exchange Formats in the U.S. National Tuberculosis Surveillance System
}

\author{
Wilfred Bonney ${ }^{\star 1,2}$, Sandy F. Price ${ }^{1}$ and Roque Miramontes ${ }^{1}$ \\ 'Data Management, Statistics and Evaluation Branch, Division of Tuberculosis Elimination, National Center for HIV/AIDS, Viral \\ Hepatitis, STD, and TB Prevention, Office of Infectious Diseases, Centers for Disease Control and Prevention, Atlanta, GA, \\ USA; ${ }^{2}$ Public Health Informatics Fellowship Program, Division of Scientific Education and Professional Development, Center for \\ Surveillance, Epidemiology, and Laboratory Services, Centers for Disease Control and Prevention, Atlanta, GA, USA
}

\section{Objective}

The objective of this presentation is to use a congruence of standardization protocols to effectively ensure that the quality of the data elements and exchange formats within the NTSS are optimal for users of the system.

\section{Introduction}

Disease surveillance systems remain the best quality systems to rely on when standardized surveillance systems provide the best data to understand disease occurrence and trends. The United States National Tuberculosis Surveillance System (NTSS) contains reported tuberculosis (TB) cases provided by all 50 states, the District of Columbia (DC), New York City, Puerto Rico, and other U.S.-affiliated jurisdictions in the Pacific Ocean and Caribbean Sea [1]. However, the NTSS currently captures phenotypic drug susceptibility testing (DST) data and does not have the ability to collect the rapid molecular DST data generated by platforms such as Cepheid GeneXpert MTB/ RIF, Hain MTBDRplus and MTBDRsl, Pyrosequencing, and Whole Genome Sequencing [2-6]. Moreover, the information exchanges within the NTSS (represented in HL7 v2.5.1 [7]) are missing critical segments for appropriately representing laboratory test results and data on microbiological specimens.

\section{Methods}

The application of the standardization protocols involves: (a) the revision of the current Report of Verified Case of Tuberculosis (RCVT) form to include the collection of molecular DST data; (b) the enhancement of the TB Case Notification Message Mapping Guide (MMG) v2.03 [8] to include segments for appropriately reporting laboratory test results (i.e., using Logical Observation Identifiers Names and Codes (LOINC) as a recommended vocabulary) and microbiology related test results (i.e., using Systematized Nomenclature of Medicine -- Clinical Terms (SNOMED CT) as a recommended vocabulary); and (c) the standardization of the laboratory testing results generated by the variety of molecular DST platforms, reported to TB health departments through electronic laboratory results (ELR), using those same standardized LOINC and SNOMED CT vocabularies in HL7 v2.5.1 [7].

\section{Results}

The application of the standardization protocols would optimize early detection and reporting of rifampin-resistant TB cases; provide a high-quality data-driven decision-making process by public health administrators on TB cases; and generate high-quality datasets to enhance reporting or analyses of TB surveillance data and drug resistance.

\section{Conclusions}

This study demonstrates that it is possible to apply standardized protocols to improve the quality of data, specifications and exchange formats within the NTSS, thereby streamlining the seamless exchange of TB incident cases in an integrated public health environment supporting TB surveillance, informatics, and translational research.

\section{Keywords}

Tuberculosis; Exchange formats; Surveillance system; LOINC; SNOMED CT

\section{Acknowledgments}

The authors acknowledge the support of Centers for Disease Control and Prevention (CDC), Northrop Grumman, TB controllers and laboratories throughout the USA who report cases to the CDC.

\section{References}

[1] Centers for Disease Control and Prevention (US), Division of Tuberculosis Elimination. Reported Tuberculosis in the United States, 2015 [Internet]. 2015 [cited 2017 Sept 19]. Available from https:// www.cdc.gov/tb/statistics/reports/2015/default.htm

[2] Davis JL, Kawamura LM, Chaisson LH, et al. Impact of GeneXpert MTB/RIF on patients and tuberculosis programs in a low-burden setting. a hypothetical trial. Am J Respir Crit Care Med. 2014 Jun 15;189(12):1551-9

[3] Helb D, Jones M, Story E, et al. Rapid detection of Mycobacterium tuberculosis and rifampin resistance by use of on-demand, near-patient technology. J Clin Microbiol. 2010 Jan;48(1):229-37

[4] Hillemann D, Rüsch-Gerdes S, Boehme C, et al. Rapid molecular detection of extrapulmonary tuberculosis by the automated GeneXpert MTB/RIF system. J Clin Microbiol. 2011 Apr;49(4):1202-5.

[5] Lin SYG, Desmond EP. Molecular diagnosis of tuberculosis and drug resistance. Clin Lab Med. 2014 Jun;34(2):297-314

[6] Lin SYG, Rodwell TC, Victor, T. C., et al. Pyrosequencing for rapid detection of extensively drug-resistant Mycobacterium tuberculosis in clinical isolates and clinical specimens. J Clin Microbiol. 2014 Feb;52(2):475-82

[7] HL7 International. HL7 Version 2.5.1 Implementation Guide: Electronic Laboratory Reporting to Public Health, Release 2 (US Realm) [Internet]. 2014 [cited 2017 Sept 19]. Available from https:// www.hl7.org/implement/standards/product brief.cfm?product id $=329$

[8] Centers for Disease Control and Prevention (US), Division of Tuberculosis Elimination. Tuberculosis Case Notification MMG v2.03 [Internet]. 2010 [cited 2017 Sept 18]. Available from https:// wwwn.cdc.gov/nndss/case-notification/historical-documentation.html

\section{*Wilfred Bonney}

E-mail: nto5@cdc.gov 